\title{
INNOVACIONES VIRTUALES PARA LA ENSEÑANZA DE POSGRADO EN TIEMPOS DE PANDEMIA
}

\author{
Angel Sánchez-Anguita Muñoz \\ Universidad de Salamanca \\ asam@usal.es \\ María Fátima Pulido López \\ Universidad de Salamanca \\ Berta Pérez López \\ Universidad de Salamanca
}

Recepción Artículo: 12 mayo 2021

Admisión Evaluación: 12 mayo 2021

Informe Evaluador 1: 18 mayo 2021

Informe Evaluador 2: 25 mayo 2021

Aprobación Publicación: 15 junio 2021

\section{RESUMEN}

Antecedentes: La pandemia que ha azotado el mundo ha provocado cambios insospechados en todos los ámbitos de la vida humana. La enseñanza ha debido adaptarse a esta nueva situación, pasando a una docencia virtual por el confinamiento. Los nuevos escenarios docentes nos muestran que tenemos que debemos estar preparados para adaptar la forma de enseñar a los nuevos medios y técnicas virtuales, basados en las TICs y que en los últimos años indican ser excelentes auxiliares de la enseñanz.. Por ello, es tan importante buscar o crear herramientas de carácter audiovisual para ser usadas desde las plataformas informáticas disponibles de forma interactiva (Sánchez-Anguita et al, 2016). Objetivos: Explorar e incorporar nuevas herramientas y recursos docentes para facilitar la adaptación a la formación virtual usando nuevos canales de formación virtual y nuevos métodos adaptados al formato audiovisual. Muestra: Se realizó prueba piloto con participantes en posgrado para valoración de utilidad de herramientas. Método: La investigación se ha realizado elaborando 0 adaptando materiales para ampliar posibilidades de recursos que facilitaran la docencia virtual. Con uso de canales virtuales perfeccionados o novedosos y herramientas como: foros, prácticas, cuestionarios, webinar, píldoras audiovisuales, videos, videoconferencias, tutorías virtuales, enlaces web, etc. Resultados: Los resultados indican que se pueden adaptar las nuevas metodologías a los nuevos formatos según el EEES para conseguir un panel de recursos didácticos adaptados a las nuevas tecnologías (Sánchez-Anguita et al.,2017). Tanto la mejora de las plataformas como Studium de la Universidad de Salamanca como los canales de comunicación han facilitado que podamos mejorar o adaptar herramientas y recursos al nuevo escenario de virtualidad. Conclusión: Valoración positiva de los diversos recursos audiovisuales por parte de los participantes, para la mejora del proceso enseñanza/aprendizaje, potenciando la adquisición de competencias transversales facilitado por el avance en TICs, una docencia más dinámica, y nuevas formas de comunicación.

Palabras clave: herramientas docentes; enseñanza virtual; pandemia 


\section{ABSTRACT}

Virtual innovations for postgraduate education in times of pandemic. Background: The pandemic that has hit the world has caused unexpected changes in all areas of human life. Teaching has had to adapt to this new situation, moving to virtual teaching due to confinement. The new teaching scenarios have shown us that we must be prepared to adapt the form of teaching to the new virtual media and techniques, based on ICTs and that in recent years have shown to be excellent teaching aids. For this reason, it is It is so important to find or create audiovisual tools to be used from the computer platforms available in an interactive way (Sánchez-Anguita et al, 2016). Objectives: Explore and incorporate new teaching tools and resources to facilitate adaptation to virtual training using new virtual training channels and new methods adapted to the audiovisual format. Sample: A pilot test was carried out with postgraduate participants to assess the usefulness of tools. Method: The research has been carried out by developing or adapting materials to expand possibilities of resources that facilitate virtual teaching. With the use of improved or innovative virtual channels and tools such as: forums, practices, questionnaires, webinars, audiovisual pills, videos, videoconferences, virtual tutorials, web links, etc. Results: The results indicate that the new methodologies can be adapted to the new formats according to the EHEA to achieve a panel of didactic resources adapted to the new technology (Sánchez-Anguita et al., 2017), both the improvement of the platforms such as Studium of the University of Salamanca and the communication channels. They have made it easier for us to improve or adapt tools and resources to the new virtuality scenario. Conclusion: Positive assessment of the various audiovisual resources by the participants, for the improvement of the teaching / learning process, promoting the acquisition of transversal skills facilitated by the advancement in ICTS, a more dynamic teaching, and new forms of communication.

Keywords: teaching tools; virtual teaching; pandemic

\section{ANTECEDENTES}

Los nuevos escenarios docentes, como los acontecidos por la pandemia del COVID-19 nos han demostrado que debemos estar preparados para adaptar la forma de enseñar a los nuevos medios y técnicas virtuales, basados en las nuevas tecnologías y que, en los últimos años, han demostrado ser excelentes auxiliares de la enseñanza. Incluso, en algunas situaciones, son las únicas posibles, como en la pandemia del coronavirus que se inició en 2020.

Según la UNESCO, más de 861.7 millones de niños y jóvenes en 119 países se han visto afectados al tener que hacer frente a la pandemia que ha ocurrido este año (Villafuerte, 2020).

El profesorado y el personal educativo ha debido enfrentar las demandas de apoyo socioemocional y de salud mental de las y los estudiantes y sus familias, dimensión que ha cobrado creciente relevancia durante la pandemia (CEPAL, 2020).

Los cambios en legislación educativa y los cambios coyunturales de carácter socioeconómico y en la actualidad, también sanitario por la pandemia, hacen necesario que los estudios universitarios deban reinventarse. Dificultades de financiación por el aumento de la competitividad, los efectos de las crisis económicas han provocado un aumento de los estudios en formato virtual o semipresenciales, primero en los estudios de posgrado y van surgiendo en los estudios de grado. Es por ello, que se hace necesario adaptar las herramientas, materiales y también la metodología a estos nuevos formatos de las materias que se imparten y que tenemos adaptadas al Espacio Europeo de Educación Superior (EEES).

La virtualidad en el COVID-19 fue vivenciada por docentes y estudiantes, como un desafío que flexibiliza los procesos de enseñanza y aprendizaje pero con inseguridad y estrés. Por el desconocimiento de estrategias didácticas adecuadas en docentes y la incertidumbre por no conocer el encuadre de la experiencia en estudiantes (Pequeño et al., 2020)

Los estudiantes, de forma mayoritaria, valoran negativamente el paso a la virtualidad, pues este se asocia, de forma recurrente, con un incremente de la carga lectiva. La teleformación ha contribuido a impactar negativamente en la visión del alumnado sobre sus formadores, mientras que estos últimos demandan competencias digitales básicas en los jóvenes universitarios (Tejedor et al., 2020) 
Las nuevas tecnologías de la información y comunicación, son herramientas tecnológicas dinámicas que en la actualidad permiten su desarrollo, mediante diferentes estrategias en los procesos de comunicación virtual entre profesores y estudiantes, que pueden ayudar a captar mejor su atención, por medio de la World Wide Web (WWW) a través del acceso a correos electrónicos, bibliotecas virtuales, chats, foros, uso de recursos digitales como Apps, videoconferencias en tres formas: conferencia, taller o curso/seminario (Juanes et al., 2020)

La sociedad red es en la que los alumnos tienen a su disposición "entornos y herramientas con las que pueden expresar y explorar sus identidades, sitios para compartir videos, blogs, videoblogs y mundos virtuales" (Gardner y Davis, 2014)

Mientras que en un aula el profesor tiene la inmediatez de la comunicación con sus alumnos, en un evento a distancia la interacción depende de conexiones, velocidad de transmisión de datos, video y audio de calidad. Estar en dos dimensiones en vez de tres es un factor de agotamiento psicológico y hay que emplear mayor esfuerzo en expresarse y en comprender al otro (Mendiola, 2020).

Hay novedosos cambios en las formas de entender la enseñanza universitaria. Hemos vivido un cambio sustancial en los objetivos, los contenidos y las metodologías de impartición de la docencia. Todo esto ha supuesto un proceso innovador, relevante en el marco de enseñanza y en el aprendizaje en el que estamos inmersos (Sánchez-Anguita et al., 2016).

El docente tiene que ser capaz de innovar, reflexionar y transformar sus propuestas didácticas para responder a las demandas sociales que vive el mundo en medio de una crisis sanitaria (Tejedor et al. 2020). Los responsables docentes debemos comprometernos con adaptar las enseñanzas universitarias a un modelo más centrado en el trabajo autónomo del alumno, en el desarrollo de sus capacidades de aprendizaje y nuevas formas competenciales, orientado por el docente, pero también con metodología hacia el trabajo supervisado en una evaluación continua, valorándose el esfuerzo individual y también trabajo de equipo, cambiando la metodología tradicional de la clase magistral basada en la mera transmisión de conocimientos por parte del docente y sólo en una evaluación final (Sánchez-Anguita et al., 2017).

Con la implantación del nuevo modelo de enseñanza universitaria que plantea el Espacio europeo para la educación superior (EEES), nos encontramos con nuevos retos y cambios profundos que nos obligan a realizar cambios en metodologías, contenidos y herramientas para la impartición de las asignaturas, estos cambios suponen una innovar tanto desde el punto de vista de la enseñanza como del propio aprendizaje en la etapa universitaria. Debemos realizar esfuerzo por cambiar nuestra forma de visualizar las enseñanzas universitarias a un modeIo más centrado en el alumno y en su potencialidad o formas de aprendizaje de nuevas competencias y habilidades, dirigido por el profesor, pero también potenciando la autonomía del aprendizaje en tiempo y espacio, a través del esfuerzo individual y grupal con el uso de nuevos canales de información y el uso de nuevas herramientas que hagan más rápido y efectivo la adquisición del conocimiento. a diferencia del marco tradicional que estaba basado más en la transmisión de conocimientos del profesor al alumno y en una prueba final como forma de evaluación global (Sánchez-Anguita et al., 2019).

Las indicaciones contenidas en los documentos del EEES hacen referencia a nuevos procedimientos y actividades innovadoras, que impliquen al alumno en la búsqueda personal del conocimiento y en la adquisición proactiva de sus propias competencias, teniendo un significado mayor del docente como orientador que como trasmisor del conocimiento. Es por ello, que los profesores debemos experimentar y usar otros medios y técnicas a nuestro alcance con las oportunidades que ofrecen las NTICs (Nuevas Tecnologías de la Información y la Comunicación). Estas tecnologías se han convertido en herramientas básicas e imprescindibles para el profesorado ofreciendo más posibilidades metodológicas tanto a nivel teórico como práctico. En los últimos tiempos han aumentado la oferta educativa en forma de cursos de formación o másteres de competencias en las NTICs. Que estas ofertas han tenido una gran aceptación siendo apreciadas por los alumnos universitarios debido a que pertenecen a una generación nacida con ellas y a la necesidad de tener varios títulos de grado y posgrado como requisito para la incorporación profesional. Todos los recursos de tipo electrónico e interactivos son conocidos 
y normalmente apreciados por el alumno. Es por ello, que los docentes deben adaptarse y hacer uso de ellos para una mejor forma de trasmitir conocimientos y que signifique un mayor aprovechamiento de las competencias y capacidades de los alumnos de las distintas áreas y sectores (Sánchez-Anguita et al., 2017).

Con las novedosas formas de formación "online" se busca que los alumnos se impliquen en la búsqueda del conocimiento por sí mismos y en la construcción proactiva de sus competencias, teniendo al docente más como guía y tutor a distancia. Por ello, es tan importante buscar y crear herramientas de carácter audiovisual para ser usados desde plataformas informáticas de forma interactiva (Sánchez-Anguita et al., 2017).

Como se indica en el Decálogo para estudiar en línea en tiempos de coronavirus y el rediseño del curso de la Universitat Oberta de Calalunya (UOC), se establece que hay que tener en cuenta la elaboración de actividades con recursos didácticos que ayuden a los estudiantes a resolver por sí mismos o crear dinámicas de interacción activa como herramientas de trabajo colaborativo (Román, 2020)

Por todo lo expuesto se ha considerado la idoneidad de realizar este estudio en el que pretendemos, la búsqueda de recursos y la creación-realización de nuevos métodos adaptados al formato audiovisual y que puedan tener un carácter interactivo para su uso a través de las redes informáticas para mejorar no sólo el aprendizaje sino también su preparación para el uso de estas tecnologías posteriormente en el mundo profesional.

Se ha hecho un esfuerzo por explorar las posibilidades que brindan los medios audiovisuales y su trasmisión a través de redes interactivas como es el caso a través de la plataforma universitaria de Studium con las mejoras y actualizaciones que se han realizado en el canal para el uso en estas enseñanzas de nivel universitario (Sánchez-Anguita et al., 2017).

\section{OBJETIVOS}

Con el uso de los nuevos canales de formación virtual pretendemos que los estudiantes se impliquen más en la búsqueda del conocimiento por sí mismos y en la construcción proactiva de sus competencias, teniendo al docente más como un guía y tutor a distancia de su aprendizaje. Con este nuevo estudio buscamos innovar, con nuevos recursos y aplicación de nuevos métodos adaptados al formato audiovisual. Que desde su carácter interactivo y su uso a través de las redes sirva para mejorar el aprendizaje y la preparación para el uso de estas tecnologías en el mundo profesional. Por ello, el objetivo general es la creación, adquisición o adaptación de nuevas metodologías y recursos para la enseñanza de los contenidos teóricos y prácticos de posgrado a una versión mejorada y virtual (Sánchez-Anguita et al., 2017).

Objetivos específicos:

- Diseñar y elaborar un programa de adaptación de recursos y métodos docentes para impartir la enseñanza en formato "online" adaptado a la EEES (Sánchez-Anguita et al., 2017).

Usar nuevos recursos audiovisuales didácticos para el aprendizaje práctico de las asignaturas del posgrado, con materiales iterativos y de simulación necesarios para su mejor aprendizaje

- Crear herramientas metodológicas innovadoras para afianzar los conocimientos e implicación en las prácticas

- Usar nuevos canales interactivos para implantar metodologías nuevas más activas en enseñanza-aprendizaje de contenidos de tipo online (Sánchez-Anguita et al., 2017).

- Adaptar nuevas formas de comunicación interactiva: foros, chats, videoconferencias, etc.

- Mejorar conocimientos y uso de nuevas tecnologías en docentes y estudiantes

En esta investigación, la hipótesis planteada es que nuevas herramientas y recursos adaptadas a la formación virtual serán aceptadas y mejorarán la enseñanza-aprendizaje de profesores y estudiantes.

\section{METODOLOGÍA}

Han participado en este estudio once profesores y treinta y cuatro estudiantes de posgrado de la Universidad de Salamanca. 
El estudio se desarrolló en cinco meses y en cinco fases (Sánchez-Anguita et al., 2019):

1a Fase: Reuniones de coordinación y puesta en común de ideas para desarrollar, entre los docentes participantes en el estudio, mediante reuniones, videoconferencias, mail o teléfono para la adecuación del trabajo a realizar.

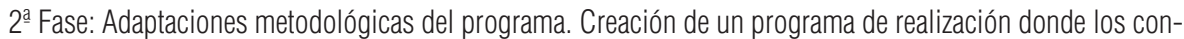
tenidos y materiales estén integrados y sirvan para la adquisición de las competencias transversales y específicas a alcanzar por el alumnado en los objetivos del título.

3 a Fase: Planificación búsqueda y preparación de recursos, guías y apoyos didácticos. Valoración de cambios y realización 0 adaptación de materiales para las prácticas del máster, e integración en el programa. para incorporar en canales virtuales a la plataforma Studium.

4ª Fase: Simulación de implementación en "prueba piloto" a través de plataforma Studium con encuesta a participantes implicados para evaluar la idoneidad y aplicabilidad.

5a Fase: Valoración de resultados y conclusiones

Los canales utilizados para implantar los recursos fueron: ordenadores, internet a través de la plataforma Studium, aplicaciones informáticas de comunicación como Google Drive, Google Meet, Team, Zoom, Blackboard, Kaltura..., y programas software como: Final Cup, Word, Excell, Power point, Adobe.

Los recursos adaptados finalmente fueron: prácticas de caso interactivas, foros-comentarios, videoconferencias/consultas, cuestionarios/tests, píldoras audiovisuales/webinar, vídeos y enlaces web, tutorías virtuales y presentaciones (ver cuadro 1)

Cuadro1: Actividades y recursos del posgrado. Fuente: Elaboración propia

\begin{tabular}{|l|l|l|l|}
\hline $\begin{array}{l}\text { Actividad } \\
\text { práctica }\end{array}$ & Carácter & $\begin{array}{l}\text { Presencialidad/preparaci } \\
\text { ón }\end{array}$ & $\begin{array}{l}\text { Individual } \\
\text { lequipo }\end{array}$ \\
\hline $\begin{array}{l}\text { Recurso: } \\
\text { Presentación } \\
\text { Máster } \\
\text { (audiovisual y } \\
\text { escrito) }\end{array}$ & Práctico & $\begin{array}{l}\text { No presencial. No } \\
\text { preparación. }\end{array}$ & $\begin{array}{l}\text { De forma } \\
\text { individual, } \\
\text { lectura y } \\
\text { visualización de } \\
\text { la grabación de } \\
\text { la presentación }\end{array}$ \\
\hline $\begin{array}{l}\text { Despacho de } \\
\text { Tutoría }\end{array}$ & $\begin{array}{l}\text { Teórico/Práctic } \\
\text { o }\end{array}$ & $\begin{array}{l}\text { No Presencial. En } \\
\text { principio, no requiere } \\
\text { preparación no presencial. }\end{array}$ & $\begin{array}{l}\text { Puede ser de } \\
\text { consultas en } \\
\text { áreas de } \\
\text { despacho “on } \\
\text { line" }\end{array}$ \\
\hline $\begin{array}{l}\text { Recurso: } \\
\text { Lectura } \\
\text { artículos, } \\
\text { capítulos de } \\
\text { libro, etc. }\end{array}$ & Práctico & $\begin{array}{l}\text { No Presencial. } \\
\text { Preparación. }\end{array}$ & $\begin{array}{l}\text { Puede ser de } \\
\text { forma individual } \\
\text { y luego poner en } \\
\text { común "on line" }\end{array}$ \\
\hline
\end{tabular}




\begin{tabular}{|c|c|c|c|}
\hline $\begin{array}{l}\text { Tarea: } \\
\text { Foros/comentari } \\
\text { os }\end{array}$ & $\begin{array}{l}\text { Teórico/práctic } \\
\text { o }\end{array}$ & $\begin{array}{l}\text { No presencial. Se hace a } \\
\text { través de la plataforma }\end{array}$ & $\begin{array}{l}\text { En grupo. Los } \\
\text { participantes } \\
\text { suben sus } \\
\text { respuestas y } \\
\text { pueden opinar o } \\
\text { contestar a otros } \\
\text { sobre el tema } \\
\text { elegido }\end{array}$ \\
\hline $\begin{array}{l}\text { Recurso: } \\
\text { Presentaciones } \\
\text { materiales por } \\
\text { temas } \\
\text { (audiovisual) }\end{array}$ & $\begin{array}{l}\text { De base teórica } \\
\text { con } \\
\text { aplicabilidad } \\
\text { práctica }\end{array}$ & $\begin{array}{l}\text { No Presencial. No requiere } \\
\text { preparación previa no } \\
\text { presencial }\end{array}$ & $\begin{array}{l}\text { La aportación del } \\
\text { alumnado suele } \\
\text { ser individual, } \\
\text { requiere visionar. }\end{array}$ \\
\hline $\begin{array}{l}\text { Recurso: } \\
\text { Diapositivas, } \\
\text { capítulos de } \\
\text { libros, apuntes. }\end{array}$ & $\begin{array}{l}\text { De base teórica } \\
\text { por temas }\end{array}$ & $\begin{array}{l}\text { No presencial. Requiere el } \\
\text { visionado por parte del } \\
\text { alumno. }\end{array}$ & $\begin{array}{l}\text { El alumno debe } \\
\text { revisar los } \\
\text { recursos y } \\
\text { analizar } \\
\text { comprensivamen } \\
\text { te el material } \\
\text { facilitado }\end{array}$ \\
\hline $\begin{array}{l}\text { Tarea: } \\
\text { Cuestionarios de } \\
\text { contenidos }\end{array}$ & $\begin{array}{l}\text { De carácter } \\
\text { teórico }\end{array}$ & $\begin{array}{l}\text { No presencial. Tipología: } \\
\text { test, preguntas cortas o a } \\
\text { desarrollar }\end{array}$ & $\begin{array}{l}\text { El alumno } \\
\text { individualmente } \\
\text { debe realizar la } \\
\text { tarea de forma } \\
\text { síncrona o } \\
\text { asíncrona según } \\
\text { el tipo de } \\
\text { cuestionario }\end{array}$ \\
\hline $\begin{array}{l}\text { Recurso: } \\
\text { Práctica } \\
\text { (inteactiva) }\end{array}$ & Práctico & No Presencial & $\begin{array}{l}\text { El alumno } \\
\text { individualmente } \\
\text { debe leer el caso } \\
\text { y buscar recursos } \\
\text { para dar solución }\end{array}$ \\
\hline $\begin{array}{l}\text { Tarea: Práctica } \\
\text { Caso/Debate }\end{array}$ & $\begin{array}{l}\text { De opinión y } \\
\text { discusión } \\
\text { práctica }\end{array}$ & $\begin{array}{l}\text { Preparación. No } \\
\text { presencial. Puede haber } \\
\text { debate }\end{array}$ & $\begin{array}{l}\text { Debe ser } \\
\text { individual inicio, } \\
\text { pero se puede } \\
\text { hacer debate } \\
\text { grupal y poner en } \\
\text { común a través } \\
\text { de red y/o } \\
\text { equipos para } \\
\text { solución grupal }\end{array}$ \\
\hline
\end{tabular}




\begin{tabular}{|c|c|c|c|}
\hline $\begin{array}{l}\text { Recurso: } \\
\text { Videos/películas }\end{array}$ & $\begin{array}{l}\text { De contenido } \\
\text { práctico de } \\
\text { entrevistas, } \\
\text { conferencias, } \\
\text { films, } \\
\text { documentales, } \\
\text { etc. } \\
\text { Información } \\
\text { del tema. }\end{array}$ & $\begin{array}{l}\text { No presencial. } \\
\text { Presentación de } \\
\text { la pieza audiovisual, del } \\
\text { tema o de la asignatura }\end{array}$ & $\begin{array}{l}\text { Individualizada } \\
\text { pero que se } \\
\text { puede hacer con } \\
\text { debate grupal } \\
\text { (foro) sobre los } \\
\text { resultados de } \\
\text { cada uno a las } \\
\text { cuestiones } \\
\text { planteadas. }\end{array}$ \\
\hline $\begin{array}{l}\text { Tarea: } \\
\text { Videos/películas }\end{array}$ & Práctico & No Presencial & $\begin{array}{l}\text { Debe ser } \\
\text { individual para } \\
\text { dar respuesta a } \\
\text { preguntas sobre } \\
\text { el recurso } \\
\text { audiovisual } \\
\text { como práctica o } \\
\text { elemento de } \\
\text { apoyo a } \\
\text { contenidos. }\end{array}$ \\
\hline $\begin{array}{l}\text { Tarea: } \\
\text { Videoconferenci } \\
\text { as }\end{array}$ & Práctico & No presencial & $\begin{array}{l}\text { Preferentemente } \\
\text { grupo o } \\
\text { individual para } \\
\text { resolución de } \\
\text { dudas. } \\
\text { Orientación } \\
\text { tareas y TFM }\end{array}$ \\
\hline $\begin{array}{l}\text { Recurso: } \\
\text { Bibliografía }\end{array}$ & Teórico & $\begin{array}{l}\text { No presencial. Requiere la } \\
\text { búsqueda de las fuentes y } \\
\text { el uso para la temática }\end{array}$ & $\begin{array}{l}\text { El uso de la } \\
\text { bibliografía } \\
\text { puede ser } \\
\text { individual o en } \\
\text { grupo. Compartir } \\
\text { recursos } \\
\text { bibliográficos en } \\
\text { foros. }\end{array}$ \\
\hline
\end{tabular}

\section{RESULTADOS}

Los resultados conseguidos con este trabajo nos indican que:

Se adaptaron las asignaturas a las nuevas metodologías de enseñanza - aprendizaje en nuevos formatos virtuales y según directrices de EEES.

Se implementaron metodologías, materiales y herramientas para la enseñanza en formato "online" de enseñanza a distancia para llegar mejor a los objetivos del máster en la adquisición y desarrollo de las principales competencias específicas y transversales.

Se aumentó el uso de plataformas de internet para la realización de tareas e interactuar entre grupo de alumnos y con sus profesores aumentando la coordinación (Sánchez-Anguita et al., 2017). 
Para la evaluación de objetivos y resultados se valoró la duración y la frecuencia de participación en foros, consultas privadas y comunicaciones interactivos a través de apartado "Novedades" de Studium. También la participación en videoconferencias, el acceso a las calificaciones y asignaturas. También la opinión de alumnos sobre: formas de evaluación, materiales y recursos, canales, contenidos, metodología y docencia.

Para la evaluación del impacto del cambio se preguntó por:

- Idoneidad de fechas, plazos de entrega tareas, fechas evaluaciones, cronograma no existiendo desacuerdo en este apartado.

- Por la utilidad de materiales y recursos para la realización de tareas prácticas, foros-comentarios o para la realización pruebas escritas sobre contenidos. Siendo bastantes considerados los enlaces web, artículos, y píldoras de contenidos.

- Conveniencia de formatos de clases en videoconferencias y a través de: Zoom, Google Meet o Blackboard, siendo valoradas, de forma similar aunque la última era más utilizada por estar incorporada en la plataforma Studium.

- Se interpreta como útil el uso de foros en novedades para notificaciones, tutorías virtuales y formas de comunicación en grupo.

Sobre el cuestionario que se aplicó a los participantes los resultados indican que:

- Los estudiantes prefieren evaluaciones asíncronas y "trabajos" mejor que cuestionarios o tests síncronos.

- Usar cuestionarios con respuestas de elección múltiple de dos o tres opciones son más aceptados por los estudiantes que los de cuatro opciones y sobre todo si se permiten 1,5 minutos o más por respuesta.

- Prefieren tener varios intentos para responder a los cuestionarios o tests.

-El acceso a calificaciones es más inmediato y reciben el feed-back de su desempeño de forma casi instantánea y personalizada.

- La novedad de foro/comentarios ha incrementado el interés sobre artículos y publicaciones actuales y la participación con opiniones en debates.

- Las prácticas con recursos y por internet son más aceptadas por los estudiantes.

- El uso de videoconferencias a través de: Blackboard y Zoom ha aumentado la participación interactiva por permitir conectarse desde cualquier sitio y fácilmente.

- Aprecian las posibilidades de grabación de: píldoras audiovisuales y videoconferencias que permiten visionar en diferido la sesión si hubo alguna dificultad de conexión.

- La participación en consultas privadas y tutorías son más rápidas con el profesor y aumentaron la comprensión para afrontar las tareas.

- Las presentaciones y despedidas audiovisuales son apreciadas y contestadas por los estudiantes.

Para la evaluación del estudio se preguntó a docentes por la valoración de las formas de reunión y número suficiente para desarrollar nuevas ideas, la búsqueda de materiales, aplicaciones y canales. La participación del grupo en el proyecto

\section{DISCUSIÓN}

Las mejoras esperadas en el aprendizaje son que los estudiantes al disponer de más medios para la realización de sus estudios y adquisición de las competencias específicas y transversales propias de asignaturas del posgrado tengan una mejora en la adquisición de nuevas formas del propio aprendizaje y de la autonomía en el manejo de la información y adquisición de competencias. Y el proceso de enseñanza-aprendizaje es más dinámico e interactivo, mediante metodologías activas y el uso de medios que potencian tanto la comunicación como la participación como videoconferencias, foros de debate, café virtual, etc. Que se mejora de la realización de trabajos de grupo 0 individuales como el Trabajo Fin de Máster mejor supervisado por el director del TFM en todo momento. Se mejora el uso de los canales de comunicación e intercambio de conocimientos de que disponen profesores y alumnos, con las mejoras aplicadas en la plataforma utilizada de Studium. 
Sobre las mejoras sobre la comunicación y la docencia: mejora en el proceso de enseñanza-aprendizaje centrado en el uso de más y mejores medios y en el trabajo autónomo del estudiante. Se pueden usar distintos formatos de evaluación síncrona 0 asíncrona. Con internet se potencia la adquisición de competencias transversales y específicas de las asignaturas mediante los casos prácticos interactivos en formato "tarea" de Studium para la solución. Se mejora del uso y dominio por parte de estudiante y profesor de los medios interactivos y las NTICs como herramientas de trabajo de grupo con datos en la nube basado en: chats, videoconferencias, notas, acceso a contenidos, etc. Aumentan y mejora de las vías de comunicación entre estudiantes y profesores, gracias al uso de herramientas interactivas basadas en plataformas de intercambio de información y enlaces.

Sobre las mejoras sobre la oferta docente: se aumentan las posibilidades de oferta docente de la universidad con la adaptación e innovación de asignaturas a la versión de enseñanza a distancia para un presente y futuro próximo de cada vez, mayor demanda de estas enseñanzas en formato "online". El alumnado dispone de más medios para la realización de sus estudios y adquisición de las competencias específicas y transversales. Se fomenta la adquisición de nuevas formas del propio aprendizaje por parte del alumnado y de la autonomía en el manejo de la información y adquisición de competencias. El proceso de enseñanza-aprendizaje es más dinámico e interactivo, mediante metodologías activas y el uso de medios que potencian tanto la comunicación como la participación, a través de trabajos individuales y en grupo. Se aumenta el uso de los canales de comunicación e intercambio de conocimientos de que disponen profesores y alumnos con la mejora de plataformas como Studium. Se mejora de la adquisición de conocimientos por parte del alumnado y de las competencias específicas y transversales propias de un máster de estas características. Se desarrollan habilidades y conocimientos por parte del alumnado para su propia organización en el manejo de la información y que potencian la reflexión como la participación, a través del trabajo "online". Se facilita el aprendizaje en el uso de nuevas herramientas de adquisición o evaluación a través de internet.

El impacto sobre la docencia es: la adquisición de competencias transversales y específicas de las asignaturas y la mejora del uso y dominio de los medios interactivos y las NTICs. Se mejoran las vías de comunicación entre estudiantes y el profesorado, gracias al uso de herramientas interactivas basadas en plataformas de intercambio de información. Se fomenta la adaptación a cambios y mejora en las formas y métodos a utilizar para lograr los objetivos docentes. Se facilita trabajar activamente sobre adquisición de competencias transversales y específicas del título y a realizar una docencia más dinámica que mejore resultados y la comunicación.

\section{CONCLUSIONES}

Se han cumplido los objetivos previstos en el estudio, dejando evidencia de que se pueden realizar nuevas formas de confeccionar herramientas y materiales 0 adaptar otras a partir de los fundamentos del EEES, pero basadas en las nuevas tecnologías y en medios audiovisuales e interactivos. Para conseguir la mejora de docentes y alumnos, haciendo aportaciones novedosas en las metodologías docentes y aumentando la eficacia en los procesos de enseñanza-aprendizaje.

Se ha conseguido el objetivo de investigar nuevas herramientas y metodologías para adaptar la enseñanza y aplicación de habilidades prácticas a un formato "online" de enseñanza a distancia a través de las nuevas tecnologías. Con estas nuevas herramientas y metodologías se consigue mejorar la comprensión y asimilación de contenidos del posgrado en Psicosalud Laboral.

Se han explorado las posibilidades que brindan los medios audiovisuales y su trasmisión a través de redes interactivas, como es el caso, a través de la plataforma universitaria de Studium con las mejoras y actualizaciones que se han realizado en el canal para el uso en estas enseñanzas de nivel universitario. Las herramientas y materiales se aplicarán en la asignatura como nuevos soportes de apoyo para la superación por parte del alumnado y quedando como experiencia para si en un futuro se pudiera impartir toda la asignatura en formato online (Sánchez-Anguita et al., 2019)

Se ha completado la búsqueda de recursos y la creación-realización de nuevos métodos adaptados al formato audiovisual y que puedan tener un carácter interactivo para su uso a través de las redes informáticas para mejo- 
rar no sólo el aprendizaje sino también su preparación para el uso de estas tecnologías posteriormente en el mundo profesional.

Se ha conseguido trabajar en equipo valorando las aportaciones que han podido aportar cada miembro al proyecto común del posgrado.

Se ha valorado como importante la incorporación de una red social como Google+ para facilitar un nuevo canal interactivo al que todos los estudiantes de la universidad tienen acceso a través de su cuenta "gmail" para poder tener una plataforma de avisos, conversaciones y foros sobre temas y materiales del curso a través de los "círculos" o grupos de participantes.

La época COVID-19 ha propiciado una concienciación sobre la necesidad de adaptar metodologías y recursos docentes a los canales virtuales en NTICs. Los resultados indican que se pueden adaptar nuevas herramientas a los nuevos formatos según el EEES para conseguir un panel de recursos didácticos adaptados a las nuevas NTICS.

Con estas nuevas herramientas y metodologías se consigue mejorar la aceptación y asimilación de contenidos del posgrado y una valoración positiva de los recursos audiovisuales usados por los participantes, para la mejora del proceso enseñanza/aprendizaje. La mejora de las plataformas como Studium y los canales de comunicación han facilitado mejorar o adaptar herramientas y recursos al nuevo escenario de virtualidad, mejorando le adquisición de competencias transversales, facilitado por el avance en NTICs, para una docencia más dinámica y el manejo de nuevas formas de comunicación.

\section{REFERENCIAS BIBLIOGRÁFICAS}

Comisión Económica para América Latina y el Caribe. (2020). La educación en tiempos de pandemia por COVID19. Informe COVID-19. CEPAL-UNESCO.

Gardner, H., y Davis, K. (2014). La generación APP. Paidós Ediciones.

Juanes, B. Munévar, O., Cándelo, H. (2020). La virtualidad en la educación. Aspectos claves para la continuidad de la enseñanza en tiempos de pandemia. Revista Conrado, 16, 448-452.

Mendiola, J. (2020). ¿Por qué nos agotan psicológicamente las videoconferencias? El País. https://elpais.com/tecnologia/2020-05-02/por-que-nos-agotan-psicologicamente-lasvideoconferencias.html.

Pequeño, I., Gadea, S., Alborés, M., Chiavone, L., Fagúndez, C., Giménez, S. y Santa Cruz, A. B. (2020). Enseñanza y aprendizaje virtual en contexto de pandemia. Intercambios, 7, 7-14.

Román, L. (2020). La educación online en la pandemia: problemas y claves para el futuro. Educación 3.0. https://www.educaciontrespuntocero.com/noticias/educacion-online-en-la-pandemia/

Sánchez-Anguita, A., De Elena, J., Conde, J. (2016). Auditoría de Recursos Humanos. Programa de Adaptación de Recursos y metodología docente a la enseñanza online de asignatura del grado de relaciones laborales y recursos humanos adaptadas al EEES. Memoria de Proyecto ID 2015/0101. Universidad de Salamanca.

Sánchez-Anguita, A., De Elena, J., González, L, Conde, J. y Calvo, R. (2017). Programa de adaptación de recursos y metodologías docentes a la enseñanza online de la asignatura del grado en relaciones laborales y recursos humanos "Gestión por competencias" adaptada al EEES. Memoria de Proyecto ID 2016/220. Universidad de Salamanca.

Sánchez-Anguita, A., De Elena, J., Conde, J. y Calvo, R. y Pulido, M. F. (2019). Programa de adaptación metodológica y de herramientas docentes para la enseñanza virtual de la materia Psicología de la Salud Laboral, del grado en relaciones laborales y recursos humanos en el ámbito del EEES. Memoria de Proyecto ID 2018/191. Universidad de Salamanca.

Tejedor, S., Cervi, L., Tusa, F. y Parola, A. (2020). Educación en tiempo de pandemia. Revista Latina, 78, 19-40.

Villafuerte, P. (2020). Educación en tiempos de pandemia: COVID-19 y equidad en el aprendizaje. Observatorio de Innovación Educativa y Tecnológico de Monterrey. https://observatorio.tec.mx/edu-news/educacion-entiempos-de-pandemia-covid19s. 